\title{
Identifiability Issues for Rotationally Invariant Arrays
}

\author{
A. Lee Swindlehurst \\ Dept. of Electrical \& Computer Engineering \\ Brigham Young University \\ Provo, Utah 84602
}

\begin{abstract}
The popular ESPRIT algorithm provides a computationally efficient approach for direction of arrival (DOA) estimation in situations where the sensor array is composed of two identical transiated subarrays. One of the key advantages of ESPRIT is that the subarrays need not be calibrated in order to obtain the DOA estimates. In this paper, the problem of DOA estimation using sensor arrays composed of two identical, uncalibrated, and rotated subarrays is considered. It is shown that, unlike ESPRIT, such rotationally invariant arrays do not provide an identifiable parameterization of the problem; i.e., unique DOA estimates are not possible without additional calibration information when more than one signal is present.
\end{abstract}

\section{Introduction}

$\mathbf{T}$ HE MAJORITY of algorithms developed for the narrowband direction of arrival (DOA) estimation problem require that the array response be completely known for each DOA. This is usually accomplished by either direct calibration in the field, or by analytical means using information about the position and response of each individual sensor (such as is done with a uniform linear array, or ULA, for example). A notable exception is the ESPRIT algorithm [1,2], which assumes instead that the sensor array is composed of two identical, translated subarrays. The key advantage of this approach is that the DOA estimates can be computed very efficiently, without knowledge of the response of the individual subarrays. Hence, an explicit calibration of the array is not required.

Several authors have proposed extensions of ESPRIT to more generally configured arrays. In [3], arrays composed of more than two identical, translated subarrays in parallel are considered. Azimuth and elevation angle estimation using arrays with identical, translated subarrays in more than one direction have been studied in $[4,5,6,7,8,9]$. In this paper, arrays composed of two identical, rotated subarrays are investigated. Circular arrays are a special case of this type of array. It will be shown that, although such rotationally invariant arrays give rise to a data model similar to the translationally invariant case, they do not in general lead to an identifiable parameterization of the DOAs. In particular, if the subarrays are uncalibrated, unique DOA estimates are not possible whenever two or more sources are present. This contrasts with ESPRIT, which always gives unique estimates provided the subarrays are unambiguous and separated by no more than a half wavelength.

In the next section, the narrowband data model and the ESPRIT algorithm are briefly described, primarily to introduce notation. Section 3 then presents the type of rotationally invariant array considered in this analysis. Note that the definition of rotationally invariant used here is not the most general definition possible. Finally, the loss of identifiability for this type of array is established in Section 4.

\section{The ESPRIT Data Model}

Under the assumption of narrowband signals, the output of an $M$-element array $\mathbf{x}(t) \in \mathbb{C}^{M}$ due to $d$ emitters can be described by

$$
\mathbf{x}(t)=\mathbf{A}(\boldsymbol{\theta}) \mathbf{s}(t)+\mathbf{n}(t),
$$

where $\mathbf{s}(t) \in \mathbb{C}^{d}$ represents the signal amplitude and phase at time $t, \mathbf{n}(t) \in \mathbb{C}^{M}$ is additive noise, and $\mathbf{A}(\boldsymbol{\theta}) \in \mathbb{C}^{M \times d}$ is the matrix of array response vectors for each source parameterized by the DOAs $\boldsymbol{\theta}=$ $\left[\theta_{1}, \cdots, \theta_{d}\right]^{T}$ :

$$
\mathbf{A}(\boldsymbol{\theta})=\left[\begin{array}{lll}
\mathbf{a}\left(\theta_{1}\right) & \cdots & \mathbf{a}\left(\theta_{d}\right)
\end{array}\right] .
$$

It can be assumed without loss of generality that the noise is spatially white, or in other words that $\mathcal{E}\left\{\mathbf{n}(t) \mathbf{n}^{*}(t)\right\}=\sigma^{2} \mathbf{I}$. There are a number of important applications for which the model of (1) is appropriate, the goal being to use several observations from the array to estimate the DOAs $\theta_{1}, \cdots, \theta_{d}$.

As mentioned above, most algorithms developed for this problem require that $\mathbf{a}(\theta)$ be known for all possible $\theta$ in order to estimate the DOAs. This, however, is 
not true of the ESPRIT algorithm. ESPRIT assumes that the $M$-element array is composed of two identical, translated, $m$-element subarrays, where $m<M \leq 2 m$ in general. For subarrays that do not overlap (i.e., share elements), $M=2 m$, though in general, $M \leq 2 m$ since overlapping subarrays are allowed. An example of a non-overlapping array that satisfies the ESPRIT requirement is depicted in Figure 1. The arrow superimposed on each array element simply indicates the absolute orientation of the element.

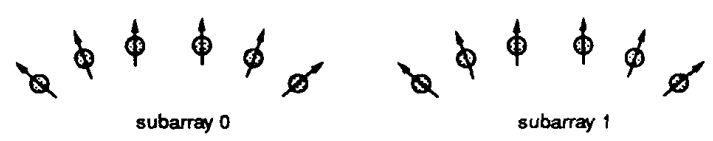

Figure 1: A Translationally Invariant Array

The assumption of translationally invariant subarrays results in a very special structure for $\mathbf{A}(\boldsymbol{\theta})$. To see this, let $\mathbf{J}_{0}$ and $\mathbf{J}_{1}$ represent the $m \times M$ selection matrices that assign the elements of $\mathbf{x}(t)$ to the two subarrays. Then

$$
\mathbf{J A}(\boldsymbol{\theta})=\left[\begin{array}{c}
\mathbf{J}_{0} \\
\mathbf{J}_{1}
\end{array}\right] \mathbf{A}(\boldsymbol{\theta})=\left[\begin{array}{c}
\mathbf{A}_{e}(\boldsymbol{\theta}) \\
\mathbf{A}_{e}(\boldsymbol{\theta}) \boldsymbol{\Phi}
\end{array}\right]
$$

where $\boldsymbol{\Phi}$ is a unitary diagonal matrix with diagonal elements $\phi_{i}$ given by

$$
\phi_{i}=\exp \left\{-j 2 \pi \Delta \sin \theta_{i} / \lambda\right\}, \quad i=1, \cdots, d,
$$

and where $\lambda$ is the wavelength of the narrowband signal, $\Delta$ is the distance between the subarrays, and $\mathbf{A}_{e}(\boldsymbol{\theta})$ represents the response of subarray 0 (assumed to be full rank $m>d$ for all $\boldsymbol{\theta}$ ).

The ESPRIT algorithm exploits the structure in (3) in the following way. If $\mathbf{E}_{s}=\left[\mathbf{e}_{1} \cdots \mathbf{e}_{d}\right] \in \mathbb{C}^{M \times d}$ represents the eigenvectors corresponding to the $d$ largest eigenvalues of the covariance $\mathbf{R}=\mathcal{E}\left\{\mathbf{x}(t) \mathbf{x}^{*}(t)\right\}$, and if no two signals are fully correlated, then it is easily shown that

$$
\boldsymbol{J} \mathbf{E}_{\boldsymbol{s}} \stackrel{\text { def }}{=}\left[\begin{array}{c}
\mathbf{E}_{0} \\
\mathbf{E}_{1}
\end{array}\right]=\left[\begin{array}{c}
\mathbf{A}_{e}(\boldsymbol{\theta}) \\
\mathbf{A}_{e}(\boldsymbol{\theta}) \boldsymbol{\Phi}
\end{array}\right] \mathbf{T},
$$

for some full rank $d \times d$ matrix $\mathbf{T}$. Solving for $\mathbf{A}_{e}(\boldsymbol{\theta})$ and substituting into the lower block of this equation leads to

$$
\mathbf{E}_{1}=\mathbf{E}_{0} \mathbf{T}^{-1} \mathbf{\Phi} \mathbf{T}=\mathbf{E}_{0} \mathbf{\Psi},
$$

where the matrix $\mathbf{\Psi}=\mathbf{T}^{-1} \mathbf{\Phi} \mathbf{T}$ has been defined. Thus, if $m \geq d$ and $\Delta<\lambda / 2$, the DOAs may be uniquely determined from the eigenvalues of the operator $\boldsymbol{\Psi}$ that maps $\mathbf{E}_{0}$ onto $\mathbf{E}_{1}$. Note that this result is independent of the value of $\mathbf{A}_{\varepsilon}(\boldsymbol{\theta})$ (as long as it is full rank), so the array need not be calibrated in order to estimate the DOAs.

Since a perfect measurement of $\mathbf{R}$ cannot be obtained, the sample covariance $\hat{\mathbf{R}}$ defined by

$$
\hat{\mathbf{R}}=\frac{1}{N} \sum_{k=1}^{N} \mathbf{x}\left(t_{k}\right) \mathbf{x}^{*}\left(t_{k}\right)
$$

is used as an estimate, and its eigenvectors $\hat{\mathbf{E}}_{0}$ and $\hat{\mathbf{E}}_{1}$ will not exactly satisfy the relationship of equation (5). Hence, there will be no operator that exactly maps the columns of $\hat{\mathbf{E}}_{0}$ onto those of $\hat{\mathbf{E}}_{1}$. Though a leastsquares estimate of $\mathbf{\Psi}$ may be easily obtained $[10,11]$, since both $\hat{\mathbf{E}}_{0}$ and $\hat{\mathbf{E}}_{1}$ have errors, a total-least-squares (TLS) [12] estimate of $\boldsymbol{\Psi}$ is more appropriate. This is the approach taken by the (TLS)-ESPRIT algorithm. As formulated in [2], the TLS estimate of $\boldsymbol{\Psi}$ is calculated by performing the singular value decomposition (SVD)

$$
\left[\begin{array}{c}
\hat{\mathbf{E}}_{0}^{*} \\
\hat{\mathbf{E}}_{1}^{*}
\end{array}\right]\left[\begin{array}{ll}
\hat{\mathbf{E}}_{0} & \hat{\mathbf{E}}_{1}
\end{array}\right]=\left[\begin{array}{ll}
\mathbf{U}_{11} & \mathbf{U}_{12} \\
\mathbf{U}_{21} & \mathbf{U}_{22}
\end{array}\right] \boldsymbol{\Sigma}\left[\begin{array}{ll}
\mathbf{U}_{11} & \mathbf{U}_{12} \\
\mathbf{U}_{21} & \mathbf{U}_{22}
\end{array}\right]^{*}
$$

and setting

$$
\hat{\mathbf{\Psi}}_{T L S}=-\mathbf{U}_{12} \mathbf{U}_{22}^{-1} .
$$

The ESPRIT DOA estimates are then calculated using the eigenvalues of $\hat{\boldsymbol{\Psi}}_{T L S}$ and equation (4). The modular nature of the algorithm (it requires successive eigendecompositions or SVDs of order $M, 2 d$, and $d)$ coupled with the fact that it requires no explicit gradient search makes it very attractive from a computational standpoint.

\section{Rotationally Invariant Arrays}

While the class of arrays for which ESPRIT is applicable is an important one, there are many other common array structures that do not possess translationally invariant subarrays. Circular arrays are a prime example. It would be desirable to develop fast algorithms like ESPRIT that exploit the regular structure present in such arrays.

This paper considers the possibility of developing an ESPRIT-like algorithm for $180^{\circ}$ rotationally invariant arrays, i.e., arrays composed of two identical rotated subarrays. To illustrate the type of array geometry to be studied, consider the sensor configurations of Figures 2 and 3. The two subarrays consist of the upper six and lower six elements, respectively, and, as 
before, the arrow superimposed on each sensor indicates its absolute orientation. If Array 1 is rotated $180^{\circ}$, and each of the sensors is forced to maintain its absolute orientation, then another array identical to Array 1 is obtained. The same is also true for Array 2 if the orientation of the individual sensors is allowed to change as the array is rotated. Strictly speaking, the analysis of this paper will only apply to a rotational invariance of the type exhibited by Array 1 . However, in many cases the individual antenna elements have response patterns that are themselves $180^{\circ}$ rotationally invariant (i.e., they are symmetric about some axis of rotation), in which case Arrays 1 and 2 are equivalent. Thus, the ideas presented below will often apply to arrays that possess the kind of rotational invariance exhibited by Array 2 as well.

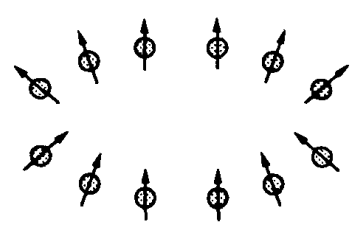

Figure 2: Rotationally Invariant Array 1

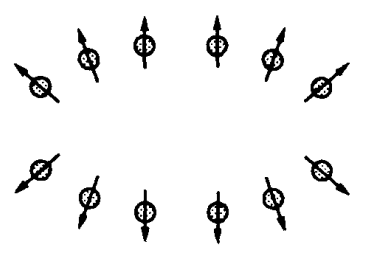

Figure 3: Rotationally Invariant Array 2

Both circular and linear arrays belong to this class. As in the case of linear arrays and translationally invariant subarrays, a circular array possesses a number of possible subarray structures that satisfy the required rotational invariance, some of which lead to overlapping subarrays. An example of a maximally overlapping subarray structure for a circular array is depicted in Figure 4.

As in the case of ESPRIT, rotationally invariant arrays like those depicted in Figures 2 and 4 lead to a very special shift structure for the array response. Instead of equation (3), vectors from the array manifold

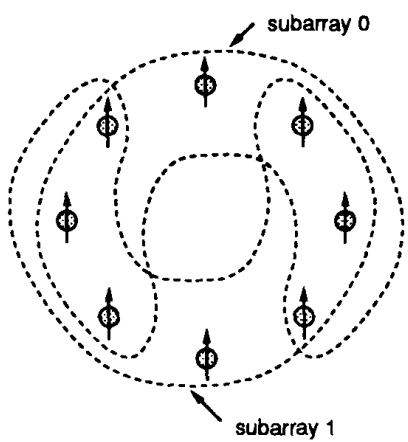

Figure 4: A Possible Subarray Configuration for a Circular Array

are described by the equation

$$
\mathbf{J} \mathbf{A}(\boldsymbol{\theta})=\left[\begin{array}{c}
\mathbf{A}_{e}(\boldsymbol{\theta}) \\
\overline{\mathbf{A}}_{e}(\boldsymbol{\theta}) \boldsymbol{\Phi}
\end{array}\right]
$$

where $\overline{\mathbf{A}}_{e}(\boldsymbol{\theta})$ denotes the complex conjugate of the subarray manifold $\mathbf{A}_{e}(\boldsymbol{\theta}), \boldsymbol{\Phi}$ is defined exactly as in (4), except that $\Delta$ is the distance between a given arbitrary sensor (the reference) and its identical twin in the companion subarray, and $\theta$ is measured with respect to the normal of the line joining these two sensors. The conjugation of $\mathbf{A}_{e}$ accounts for the fact that the propagation of a wavefront across one of the subarrays can be considered to be equivalent to the same wavefront propagating backwards across the rotated companion subarray. The diagonal matrix $\Phi$ takes care of the bulk phase shift due to the delay in propagation between the subarrays.

This particular definition forces the row of $\mathbf{A}_{\boldsymbol{e}}(\boldsymbol{\theta})$ corresponding to the reference sensor to be purely real, but this is not a problem since any non-zero phase response for the reference sensor in direction $\theta_{i}$ can be lumped together with the phase of the $i^{\text {th }}$ signal waveform. For simplicity, in this paper it will be assumed (without loss of generality) that the reference sensor corresponds to the first row of $\mathbf{A}_{e}(\boldsymbol{\theta})$, and that this row is composed of all 1's.

For an array satisfying (10), the eigenvectors corresponding to the $d$ largest eigenvalues of the covariance $\mathbf{R}$ will satisfy (compare with equation (5))

$$
\mathbf{J E}_{\boldsymbol{s}} \stackrel{\text { def }}{=}\left[\begin{array}{l}
\mathbf{E}_{0} \\
\mathbf{E}_{1}
\end{array}\right]=\left[\begin{array}{c}
\mathbf{A}_{e}(\boldsymbol{\theta}) \\
\overline{\mathbf{A}}_{e}(\boldsymbol{\theta}) \boldsymbol{\Phi}
\end{array}\right] \mathbf{T}
$$

for some full rank $d \times d$ matrix $\mathbf{T}$, and the corresponding relationship between $\mathbf{E}_{0}$ and $\mathbf{E}_{1}$ is

$$
\mathbf{E}_{1}=\overline{\mathbf{E}}_{0} \overline{\mathbf{T}}^{-1} \boldsymbol{\Phi} \mathbf{T}
$$




$$
=\overline{\mathbf{E}}_{0} \mathbf{\Psi}_{c} \text {. }
$$

The factorization $\mathbf{\Psi}_{c}=\overline{\mathbf{T}}^{-1} \mathbf{\Phi} \mathbf{T}$ is referred to as a coneigendecomposition, and the DOAs are thus a function of the coneigenvalues of $\Psi_{c}$ (e.g., see [13]). It is interesting to note that, while the eigenvalues of the ESPRIT operator $\boldsymbol{\Psi}$ are uniquely defined, the coneigenvalues of a given matrix are not. This is fundamentally the reason why unique DOA estimates are not possible when $d>1$, as demonstrated below.

\section{Identifiability}

If there is only one source present, $d=1$ and $\mathbf{E}_{s}$ is $M \times 1$. The lone element of $\Phi$ (described by (4)) in this case can be obtained by first normalizing $\mathbf{J} \mathbf{E}_{s}$ so that its first element is one, and then solving, for example,

$$
\phi=\frac{\overline{\mathbf{E}}_{1}^{*} \mathbf{E}_{0}}{\mathbf{E}_{0}^{*} \mathbf{E}_{0}} .
$$

If $\Delta<\lambda / 2$, the DOA can be uniquely determined using (4).

To demonstrate that the model of (11) is not identifiable for $d>1$, it will be shown that there exists a full rank $d \times d$ matrix $\mathbf{T}_{1}$, a full rank $m \times d$ matrix $\mathbf{A}_{1}$ with first row all 1's, and a diagonal matrix $\boldsymbol{\Omega}=\operatorname{diag}\left\{e^{j \omega_{1}}, \cdots, e^{j \omega_{d}}\right\}$ such that

$$
\left[\begin{array}{c}
\mathbf{A}_{e} \\
\overline{\mathbf{A}}_{e} \mathbf{\Phi}
\end{array}\right]=\left[\begin{array}{c}
\mathbf{A}_{1} \\
\overline{\mathbf{A}}_{1} \boldsymbol{\Omega}
\end{array}\right] \mathbf{T}_{1}
$$

for arbitrary $\omega_{1}, \cdots, \omega_{d}$ satisfying

$$
\omega_{i} \neq \omega_{k}, i \neq k, \quad \text { and }\left|\omega_{i}\right|<\pi, i, k=1, \cdots, d .
$$

In other words, there are an infinite number of matrices of the form (10) that satisfy the subspace relationship of (11), and hence there are an infinite number of possible DOA estimates given only $\mathbf{J E}_{s}$.

For a given $\boldsymbol{\Omega}$ satisfying (14), let $\mathbf{A}_{1}=\mathbf{A}_{e} \mathbf{T}_{1}^{-1}$ and $\mathbf{T}_{1}=\Omega^{-\frac{1}{2}} \mathbf{X} \boldsymbol{\Phi}^{\frac{1}{2}}$ for some real-valued $d \times d$ matrix $X$. It is easily verified that these choices satisfy equation (13). It now remains to choose $\mathbf{X}$ such that the first row of $\mathbf{A}_{1}$ is all 1's, i.e.,

$$
\left[\begin{array}{lll}
1 & \cdots & 1
\end{array}\right]=\left[\begin{array}{lll}
1 & \cdots & 1
\end{array}\right] \Omega^{-\frac{1}{2}} \mathbf{X} \boldsymbol{\Phi}^{\frac{1}{2}} .
$$

This is equivalent to choosing $\mathbf{X}$ such that

$$
\mathbf{X}^{T}\left[\begin{array}{ll}
\omega_{r} & \omega_{i}
\end{array}\right]=\left[\begin{array}{ll}
\phi_{r} & \phi_{i}
\end{array}\right]
$$

where

$$
\boldsymbol{\omega}_{r}=\operatorname{Re}\left(\operatorname{diag}\left\{\boldsymbol{\Omega}^{-\frac{1}{2}}\right\}\right)
$$

$$
\begin{aligned}
& \boldsymbol{\omega}_{i}=\operatorname{Im}\left(\operatorname{diag}\left\{\Omega^{-\frac{1}{2}}\right\}\right) \\
& \phi_{r}=\operatorname{Re}\left(\operatorname{diag}\left\{\boldsymbol{\Phi}^{-\frac{1}{2}}\right\}\right) \\
& \phi_{i}=\operatorname{Im}\left(\operatorname{diag}\left\{\boldsymbol{\Phi}^{-\frac{1}{2}}\right\}\right),
\end{aligned}
$$

$\operatorname{Re}\{\cdot\}$ and $\operatorname{Im}\{\cdot\}$ denote real and imaginary parts respectively, and $\operatorname{diag}\{\cdot\}$ here denotes the column vector formed from the diagonal elements of the argument. Provided $d \geq 2$ and the elements of $\Omega$ satisfy (14), such an $X$ is always possible since both $\left[\begin{array}{ll}\omega_{r} & \omega_{i}\end{array}\right]$ and $\left[\begin{array}{ll}\phi_{r} & \phi_{i}\end{array}\right]$ are $\operatorname{rank}^{1} 2$. Consequently, the angles $\omega_{1}, \cdots, \omega_{d}$ are equally as valid as $\phi_{1}, \cdots, \phi_{d}$ in satisfying the subspace model of (11).

\section{Conclusion}

Although the shift structure of the steering vectors for an array composed of identical rotated subarrays bears a striking resemblance to that for ESPRIT, unique DOA estimates are not possible when more than one signal is present. This loss of identifiability could only be overcome by somehow incorporating information about the individual subarray responses. However, doing so would probably mean that a computationally efficient solution such as that for ESPRIT would not be possible.

\section{References}

[1] A. Paulraj, R. Roy, and T. Kailath, "A Subspace Rotation Approach to Signal Parameter Estimation", Proceedings of the IEEE, pages 1044-1045, July 1986 .

[2] R. Roy and T. Kailath, "ESPRIT - Estimation of Signal Parameters via Rotational Invariance Techniques", IEEE Trans. on ASSP, 37(7):984995 , July 1989.

[3] A. Swindlehurst, B. Ottersten, R. Roy, and T. Kailath, "Multiple Invariance ESPRIT", to appear in IEEE Trans. on Sig. Proc., 40, April 1992.

[4] D. V. B. Rao and S. Y. Kung, "A State Space Approach for the 2-D Harmonic Retrieval Problem", In Proc. IEEE ICASSP, pages 4.10.14.10.4, 1984.

\footnotetext{
${ }^{1}$ The matrix $\left[\begin{array}{ll}\phi_{r} & \phi_{i}\end{array}\right]$ is rank 2 since the elements of $\Phi$ can be assumed to satisfy (14). If this were not true, unique DOA
estimates would not be possible even if equation (13) were not considered.
} 
[5] M. Zoltowski and D. Stavrinides, "Sensor Array Signal Processing via a Procrustes Rotations Based Eigenanalysis of the ESPRIT Data Pencil", IEEE Trans. on ASSP, 37(6):832-861, June 1989.

[6] A. Swindlehurst and T. Kailath, "2-D Parameter Estimation Using Arrays with Multidimensional Invariance Structure", In Proc. $23^{\text {rd }}$ Asilomar Conference of Signals, Systems, and Computers, pages 950-954, Asilomar, CA., November 1989.

[7] R. Johnson and G. Miner, "An Operational System Implementation of the ESPRIT DF Algorithm", IEEE Trans. on AES, 27(1):159-166, January 1991.

[8] A. Swindlehurst and T. Kailath, "Algorithms for Azimuth/Elevation Direction Finding Using Regular Array Geometries", to appear in IEEE Trans. on AES, 1992.

[9] P. Ober, E. Deprettere, and A. van der Veen, "Efficient Methods to Compute Azimuth and Elevation in High Resolution DOA Estimation", In Proc. ICASSP '91, pages 3349-3352, 1991.

[10] R. Roy, A. Paulraj, and T. Kailath, "ESPRIT A Subspace Rotation Approach to Estimation of Parameters of Cisoids in Noise", IEEE Trans. on ASSP, 34(4):1340-1342, October 1986.

[11] S.Y. Kung, C.K. Lo, and R. Foka, "A Toeplitz Approximation Approach to Coherent Source Direction Finding", In Proc. ICASSP 86, Tokyo, Japan, 1986.

[12] G. H. Golub and C. F. Van Loan, Matrix Comptutations, Johns Hopkins University Press, Baltimore, MD., 1984.

[13] R. A. Horn and C. A. Johnson, Matrix Analysis, Cambridge University Press, Cambridge, England, 1985. 